\title{
Ambulatory Urodynamic Findings Change Patient Outcomes
}

\author{
Richard G. Axell, ${ }^{1}$ Vahit Guzelburc, ${ }^{2}$ Habiba Yasmin, ${ }^{1}$ Bogdan Toia, ${ }^{2}$ Mahreen H. Pakzad, ${ }^{2}$ Rizwan Hamid, ${ }^{2}$ \\ Jeremy L. Ockrim, ${ }^{2}$ Tamsin J. Greenwell $₫ 2$ \\ ${ }^{1}$ Medical Physics and Bioengineering, UCLH NHS Foundation Trust, London, United Kingdom \\ ${ }^{2}$ Department of Urology, UCLH NHS Foundation Trust, London, United Kingdom \\ This work was performed at UCLH NHS Foundation Trust, London, United Kingdom
}

\section{Abstract}

Objectives Whilst ambulatory urodynamics (aUDS) may be used as a second-stage test for patients with refractory lower urinary tract symptoms (LUTS) having non-diagnostic conventional urodynamics (UDS), the evidence for their use is limited. We have assessed the diagnostic utility and consequent symptomatic outcome of aUDS in patients with refractory LUTS.

Methods A retrospective review of a prospectively acquired urodynamics database was made of 84 consecutive patients (23 male) with a median age 50.5 years (range 18 to 79 ) having aUDS following non-diagnostic or contradictory baseline UDS over a 12-month period. Patient demographics and urodynamic and clinical diagnosis before and after aUDS were recorded. Forty-six patients (55\%) had formal urinary symptom assessment recorded before and a minimum of 6 months following aUDS-related change in management.

Results Eighty-two patients (98\%) had a urodynamic diagnosis made following aUDS, 57(68\%) of whom had detrusor overactivity (DO); the final 2 patients had no abnormalities detected on aUDS. Change in primary UDS diagnosis occurred in 66 patients (79\%). Of these 66 patients, $59(89 \%)$ also had their clinical diagnosis changed, and $55(83 \%)$ had their management pathway changed. There was a significant improvement in urinary symptoms 6 months following aUDS.

Conclusion Change in primary diagnosis following aUDS led to a significant change in treatment care pathway and resulted in significant improvement in urinary symptoms.

\section{Introduction}

Conventional urodynamics (UDS) is considered to be the gold standard investigation for lower urinary tract symptoms (LUTS)[1,2]. Conventional UDS use rapid bladder filling and are performed in an unnatural environment. In a significant sub-group of patients, up to $54 \%$ to $56 \%$ of conventional UDS studies are unable to provide a urodynamic diagnosis that correlates with the patient's presenting LUTS[3,4]. Ambulatory urodynamics (aUDS) is recognised by the International Continence Society (ICS) as an important second-line diagnostic tool for providing a definitive diagnosis in patients who have previously had a non-diagnostic or symptomatically contradictory conventional UDS[5]. In contrast to conventional UDS, aUDS allows for natural (orthograde) bladder filling in a more natural environment, with the patient able to undertake relatively normal daily activities away from the UDS suite. aUDS also allow the

\section{Key Words}

Lower urinary tract symptoms, overactive bladder, urinary leakage, detrusor overactivity, urge urinary incontinence, stress urinary incontinence, voiding dysfunction, ambulatory urodynamics

\section{Competing Interests}

None declared.

\section{Article Information}

Received on May 6, 2021

Accepted on July 31, 2021

Soc Int Urol J.2021;2(6):354-361

DOI: 10.48083/MHMI1178 


\section{Abbreviations \\ aUDS ambulatory urodynamics \\ DO detrusor overactivity \\ ICS International Continence Society \\ LUTS lower urinary tract symptoms \\ OAB overactive bladder \\ SUl stress urinary incontinence \\ UDS urodynamics \\ UUI urge urinary incontinence \\ vUDS video-urodynamics}

patient to perform activities they know will provoke their most troubling urinary symptoms and improve the likelihood of a diagnostic test. They are, however, both time and personnel intensive and hence more costly than routine urodynamics, with limited availability in general urological practice.

We aimed to determine the diagnostic value of aUDS in patients with refractory LUTS of unknown cause following non-diagnostic or symptomatically contradictory conventional UDS (filling cystometry and pressure flow studies +/- video) and to assess if a change in patient diagnosis and/or treatment following aUDS led to a symptomatic improvement in patients.

\section{Methods and Methods}

\section{Study Population}

Eighty-four consecutive patients (23 male) having aUDS at our tertiary referral centre between 1 January 2015 and 31 December 2015 were identified from our prospectively acquired urodynamic database and their records retrospectively reviewed. This time period was chosen to allow sufficient time to evaluate diagnostic outcomes, change in treatment, and treatment outcomes. The median age of the patients was 50.5 years (range 18 to 79 ). Forty-six unselected patients (55\%) had formal assessment of their urinary symptoms recorded before and at a minimum of 6 months following aUDS. The remaining 38 patients did not have complete symptomatic follow-up data available for review. All patients had previously had conventional filling cystometry and pressure flow studies $(n=11)$ or video-urodynamics (vUDS) $(n=73)$. aUDS was performed when conventional UDS were non-diagnostic $(\mathrm{n}=36)$ or when the conventional UDS diagnosis was contradictory to the patients' major presenting symptoms (Table 1).

All patients before proceeding to simple urodynamics (filling cystometry and pressure flow studies) had received (as appropriate) lifestyle advice, continence therapist input with respect to bladder training + /- pelvic floor muscle exercise, and medications (as indicated by clinical diagnosis). Those progressing to ambulatory urodynamics wished to consider more invasive treatments for their symptoms, and it is a requirement of our National Health Service (NHS) system and National Institute for Health and Care Excellence (NICE) guidance that a urodynamic diagnosis is made prior to these more invasive treatments.

\section{Urodynamics Procedures}

aUDS studies were performed in accordance with the ICS guidelines test protocol[ $[1,5]$ using the MMS Solar LUNA module (Medical Measurement Systems, Gladbeck, Germany) and a fluid filled catheter system. A flow rate and post-void residual and urinalysis were performed before the test. After residual urine was measured, a 4.5fr bladder catheter (Mediplus 5716, Wycombe, UK) and a 4.5fr rectal balloon catheter (Mediplus 5410, Wycombe, $\mathrm{UK})$ were inserted for the measurement of intra-vesical and abdominal pressures respectively. A conductance leak pad sensor (Digitimer Pe-Que Sensor Pad, Welwyn Garden, UK) was used in all patients reporting symptoms of urinary incontinence. After zeroing the fluid filled pressure measurement transducers and flushing the measurement lines with saline, a cough was used to ensure good cancellation and accurate pressure measurement readings. The patients were advised on the use of the LUNA module events buttons to mark urgency and leakage and to activate the flowmeter to record voiding (Figure 1). As per the ICS guidelines, each patient was advised to drink $1 \mathrm{~L}$ of water over the first hour and to delay micturition as long as possible. During studies the patients were encouraged to perform activities/ manoeuvres known to be provocative for their typical LUTS. This included coughing, walking, climbing stairs, performing star jumps, going from a seated to a standing position, listening to running water, and hand washing. Patients were reviewed by the urodynamicist on an hourly basis to ensure patient compliance and the accuracy of the pressure measurement readings. Studies typically lasted between 2 and 4 hours depending on the time taken to demonstrate a urodynamic cause for the patient's most troubling urinary symptom. Tests were continued until patient's symptoms were reproduced in all studies.

All conventional UDS, vUDS, and aUDS studies were analysed by an experienced urodynamicist in accordance with the ICS guidelines, and BOO was determined in males with the Abrams-Griffiths nomogram[6] and in females with the SolomonGreenwell nomogram[7]. The results were subsequently reviewed at a multidisciplinary team (MDT) meeting to ensure accuracy of diagnosis and to determine treatment options.

\section{Statistical Analysis}

Data are expressed as mean \pm standard deviation and $P$-values were calculated using a 2 -tailed unpaired Student 


\section{TABLE 1.}

Change in primary urodynamic diagnosis following ambulatory urodynamic assessment

\begin{tabular}{|c|c|c|c|c|c|c|c|c|c|c|c|c|}
\hline \multirow{2}{*}{$\begin{array}{l}\text { Primary Baseline } \\
\text { Urodynamic } \\
\text { Diagnosis } \\
\text { (Total) }\end{array}$} & \multicolumn{11}{|c|}{ Primary Ambulatory Urodynamic Diagnosis } & \multirow{2}{*}{$\begin{array}{c}\text { Change in } \\
\text { Diagnosis } \\
N(\%)\end{array}$} \\
\hline & Acon & BOO & DSD & Нуро & IDO & LOC & Normal & $\begin{array}{l}\text { Red } \\
\text { Cap }\end{array}$ & SU & SUI & Other & \\
\hline Acon (1) & & & & & & & & & 1 & & & $1(100)$ \\
\hline B00 (9) & & 2 & & & 4 & & 1 & & & 2 & & $7(78)$ \\
\hline DSD (1) & & & 1 & & & & & & & & & $0(0)$ \\
\hline Hypo (2) & & & & & 1 & & & & 1 & & & $2(100)$ \\
\hline IDO (11) & & & & & 11 & & & & & & & $0(0)$ \\
\hline $\mathrm{LOC}(2)$ & & & & & 2 & & & & & & & $2(100)$ \\
\hline Normal (36) & & 2 & & 1 & 23 & & 1 & & 2 & 4 & 3 & $35(98)$ \\
\hline Red cap (4) & & & & & 2 & & & 1 & 1 & & & $3(75)$ \\
\hline SU (10) & & & & 1 & 8 & & & & 1 & & & $9(90)$ \\
\hline SUI (8) & & & & & 6 & & & & & 1 & 1 & 7 (89) \\
\hline Other (0) & & & & & & & & & & & & NA \\
\hline Total N (\%) & 0 & 4 & 1 & 2 & 57 & 0 & 2 & 1 & 6 & 7 & 4 & $84(100)$ \\
\hline
\end{tabular}

Acon: acontractile; B00: bladder outflow obstruction; DSD: detrusor sphincter dyssynergia; Hypo: hypocontractile; IDO: idiopathic detrusor overactivity; LOC: loss of compliance; Red cap: reduced capacity; SU: sensory urgency.

\section{FIGURE 1.}

LUNA ambulatory module

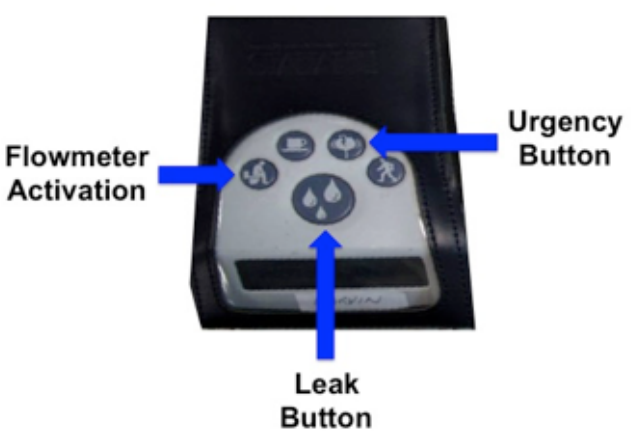

t-test for pairwise comparisons of parametric data, unless otherwise stated. Categorical data are expressed as number (percentage) and compared with the Fisher exact test. $P<0.05$ was considered statistically significant. Analysis was performed using SigmaPlot 12.5 (Systat Software Inc, San Jose CA) statistical analysis package.

\section{Results}

Following aUDS, all studies were evaluable, and a definitive urodynamic diagnosis was made in $98 \%$ $(n=82)$ of patients, $68 \%(n=57)$ of whom had detrusor overactivity (DO) (Figure 2). The 2 remaining patients (2\%) were diagnosed as having normal bladder function. A change in the primary UDS diagnosis occurred in $79 \%(\mathrm{n}=66)$ of patients following aUDS, as detailed in Table 1. Of these 66 patients $89 \%(n=59)$ also had their clinical diagnosis changed and $83 \%(n=55)$ subsequently had their management changed (Figure 3). "Clinical diagnosis" is the working diagnosis made after taking a full history, examining the patient, and performing simple tests such a MSSU, blood tests, flow rate, and postvoid residual assessment.

Of the remaining 18 patients for whom aUDS did not change their initial UDS diagnosis following conventional or vUDS, change in clinical diagnosis occurred in 10 (56\%), all of whom had their management changed. Management was also changed in $4(50 \%)$ of 
the 8 patients with no change in their UDS or clinical diagnosis following aUDS (Figure 3 ).

Overall, by providing a definitive urodynamic diagnosis in $98 \%$ of the patients, aUDS led to change in clinical diagnosis in $82 \%(n=69)$ of patients and a change in management in $82 \%(n=69)$ of patients, as detailed in Table 2.

\section{Sub-Group Analysis of Symptoms Pre- and Post- AMB UDS}

Of the 46 unselected patients (55\%) who had $\geq 6$ months symptomatic follow-up data, change in clinical diagnosis and management following aUDS led to a statistically significant improvement in their symptoms of daytime frequency, nighttime frequency, urgency, urge urinary incontinence (UUI), stress urinary incontinence (SUI), urinary incontinence of unknown cause (not UUI or SUI), poor flow, and strain void (Table 3). There was a significant improvement in ICIQ-OAB (130 \pm 35 versus $55 \pm 70, P<0.001)$ and ICIQ-SU $(15.1 \pm 9.6$ versus $7.2 \pm 10.1, P<0.001)$ scores following the changes to clinical diagnosis and management.

\section{Symptoms as a Predictor of Definitive UDS Diagnosis}

The patients' presenting symptoms were non-specific and were not significantly different between UDS diagnostic categories following aUDS (Table 4).

\section{Discussion}

We have demonstrated that aUDS is an extremely useful diagnostic tool with a $98 \%$ definitive diagnosis rate. Following aUDS, a change in urodynamic diagnosis was made in $79 \%$, a change in clinical diagnosis in $70 \%$, and
FIGURE 2.

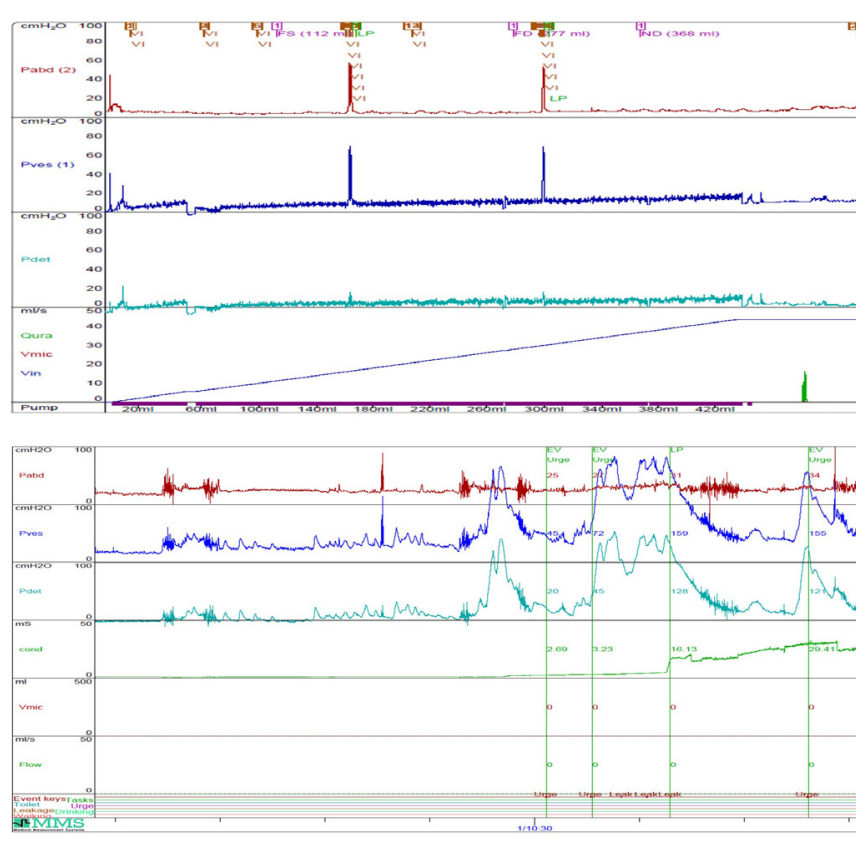

A 47-year-old female patient presenting with frequency, urgency, flooding incontinence episodes ( 3 to 5 pads per day) (a) Essentially normal filling phase urodynamics study with small leaks demonstrated on coughs $11 \mathrm{~mL}$ to $2 \mathrm{~mL})$ on vUDS. (b) DO (pp137 $\left.\mathrm{cmH}_{2} \mathrm{O}\right)$ with associated urgency and large volume UUI was demonstrated on aUDS.

a change in treatment in $75 \%$. All patients felt ambulatory urodynamics was worth their while. They were all fully informed and had provided consent for the ambulatory urodynamics tests. Their motivation was wishing to

\section{FIGURE 3.}

Change in urodynamic diagnosis, clinical diagnosis and management following aUDS

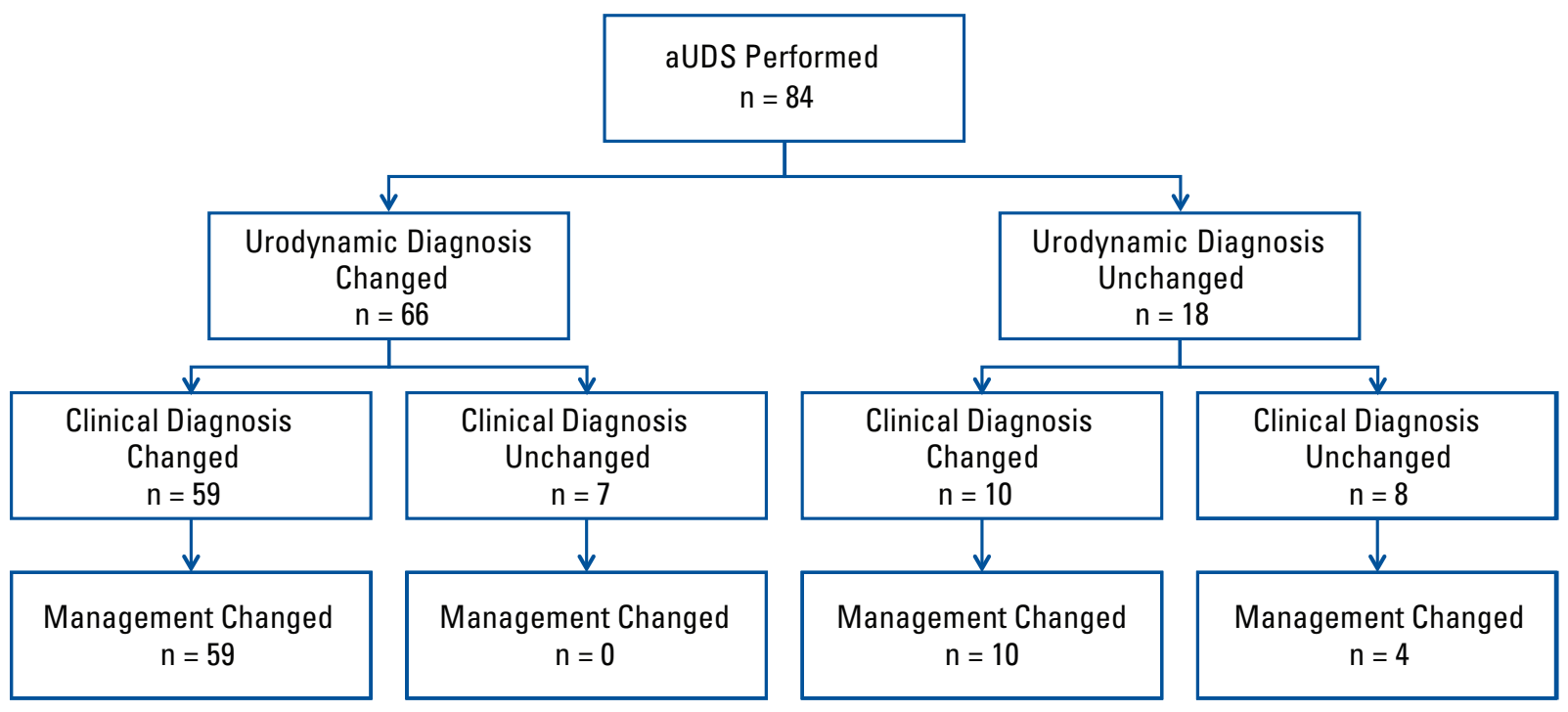




\section{TABLE 2.}

Changes in patient management following aUDS

Changes in patient management following aUDS

Intravesical botulinum toxin injection

Sacral neuromodulation

Percutaneous tibial nerve stimulation

Combined medical therapy after refusing surgical intervention

Continued conservative management after refusing surgical intervention

No intervention after normal urodynamic function confirmed

Cognitive behavioural therapy for sensory urgency syndrome

\section{5}

Reduced fluid intake after refusing surgical intervention

Clean intermittent self-catheterisation after refusing surgical intervention

\section{7}

Bladder neck incision

1

Urethral dilatation

Rectus facia sling

\section{1}

Artificial urinary sphincter

2

Milking bulbar urethra

Vesicovaginal fistula repair*

1

Clam cystoplasty

* The fistula repair was in a patient with a previous history of fistula repair (elsewhere), negative imaging, negative cystoscopy, and methylene blue test, normal simple urodynamics and ambulatory urodynamics indicating an ongoing continuous low volume leakage not related to abdominal or detrusor pressure changes. After full discussion of all options, the patient elected to have a repeat vaginal repair with complete separation of the bladder from the vagina, closure of any small fistula (none were seen), and interposition of a Martius labial fat pad flap, with resolution of her symptoms.

have a definitive diagnosis and perhaps more effective treatment of their urological issues following a "normal" simple urodynamic study.

All aUDS in this study were evaluable, which is much higher than the $74 \%$ evaluable aUDS studies reported by Pannek and Pieper[8] but similar to the $97 \%$ rate reported by Gorton and Stanton[9]. Patient symptoms were reproduced in $100 \%$ of the aUDS studies, and a urodynamic diagnosis was made in $98 \%$. This is higher than the $72 \%$ rate reported by Pannek and Pieper[8]. the $74 \%$ reported by Cantu et al.[10], and the $77.3 \%$ in women with UI by Dokmeci[4] Our unit performs 80 to 100 aUDS per year, more than in either of the studies detailed, and therefore has significant experience in performing and interpreting aUDS, which may account for the $98 \%$ diagnosis rate. However, the lower diagnosis rate seen in the Pannek and Pieper[8] study may also be related to their patient group and/or advances in the technical aspects of aUDS since 2008. Additionally, whilst both Dokmeci[4] and Pannek and Pieper[8] relied on the patient pressing the leak event marker to indicate an episode of UI, both the aUDS system of Gorton and Stanton[9] and that used in this study incorporated a leak pad sensor, which allows for definitive diagnosis of a true episode of UI.

aUDS has been shown to have a higher diagnostic yield than conventional/ vUDS. Radley et al. demonstrated this in 106 women presenting with symptoms of overactive bladder (OAB); DO was detected in 32 and 70 women on vUDS and aUDS, respectively; ie, DO was missed in up to $54 \%$ of women presenting with $\mathrm{OAB}$ on conventional UDS when compared with aUDS[3]. Dokmeci et al. showed aUDS detected the underlying pathophysiology in $77 \%$ of women presenting with urinary incontinence as compared with only $6.8 \%$ of women on conventional UDS[4]. While no cases of urodynamic SUI were demonstrated on conventional UDS in their study, $56 \%$ of patients 


\section{TABLE 3.}

Patients presenting urinary symptoms pre UDS and 6 months post change in management following aUDS

\begin{tabular}{|l|c|c|c|}
\hline Urinary Symptom & $\begin{array}{c}\text { Pre-Simple } \\
\text { UDS } \\
\text { N = 46 (\%) }\end{array}$ & $\begin{array}{c}\text { 6 Months } \\
\text { Post Change in } \\
\text { Management } \\
\text { Consequent to } \\
\text { aUDS } \\
\text { N = 46 (\%) }\end{array}$ & P value \\
\hline Day frequency & $28(61)$ & $11(24)^{*}$ & 0.0005 \\
\hline Night frequency & $25(54)$ & $10(22)^{*}$ & 0.0021 \\
\hline Urgency & $28(61)$ & $11(24)^{*}$ & 0.0005 \\
\hline UUI & $18(39)$ & $8(17)^{*}$ & 0.0344 \\
\hline SUI & $14(30)$ & $4(9)^{*}$ & 0.0158 \\
\hline Ul of unknown cause & $3(7)$ & $2(4)$ & 1.0 \\
\hline Poor flow & $13(29)$ & $2(4)^{*}$ & 0.0034 \\
\hline Strain void & $11(24)$ & $2(4)^{*}$ & 0.0137 \\
\hline Pain on filling & $3(7)$ & $1(2)$ & 0.6162 \\
\hline Pain on voiding & $5(11)$ & $0(0)^{*}$ & 0.0554 \\
\hline
\end{tabular}

had SUI demonstrated on aUDS[4]. This diagnostic rate was similar to the $77 \%$ diagnostic rate reported following aUDS for women with normal conventional UDS presented by Patravali[11]. Conversely, Robertson et al.[12] demonstrated no significant difference in the proportion of men diagnosed with BOO with simple UDS compared with aUDS; although aUDS was more sensitive for diagnosing DO. Rademakers et al.[13] have shown aUDS to be particularly useful in determining true acontractility and DO, and assigning a UDS cause for UI of unknown cause. Drossaerts et al. have also shown that aUDS is a valuable tool when assessing the effectiveness of sacral neuromodulation in patients with LUTS[14]. Likewise, we found aUDS particularly useful in diagnosing detrusor overactivity, stress urinary incontinence, and sensory urgency.

Because of the complexity of performing and reporting aUDS, it may best be concentrated in specialist centres. aUDS must be performed by an experienced urodynamicist (doctor, clinical scientist, or nurse specialist) following a strict test protocol. Conventional and vUDS are performed with the urodynamicist in the same room as the patient observing the entire test, allowing them to identify measurement artefacts and directly relate provocations to the UDS finding. This is not possible during aUDS where the patient leaves the

\section{TABLE 4.}

Symptoms as a predictor of definitive UDS diagnosis

\begin{tabular}{|c|c|c|c|c|c|}
\hline \multirow{2}{*}{$\begin{array}{l}\text { Urodynamic } \\
\text { Diagnosis }\end{array}$} & \multicolumn{4}{|c|}{ Symptoms, N (\% of Urodynamic Diagnosis Category) } & \multirow{2}{*}{$\begin{array}{c}\text { Total, } \\
\text { n (\% of total } \\
\text { urodynamic } \\
\text { diagnosis) }\end{array}$} \\
\hline & $\begin{array}{l}\text { Frequency } \\
(\mathrm{n}=91)\end{array}$ & $\begin{array}{l}\text { Urgency } \\
(n=105)\end{array}$ & $\begin{array}{l}\text { Urge Incontinence } \\
\qquad(n=76)\end{array}$ & $\begin{array}{l}\text { Stress Incontinence } \\
\qquad(\mathrm{n}=47)\end{array}$ & \\
\hline Detrusor overactivity & $43(70)$ & $53(87)$ & $43(70)$ & $22(26)$ & $61(48)$ \\
\hline $\begin{array}{l}\text { Urodynamic stress } \\
\text { urinary incontinence }\end{array}$ & $15(75)$ & $16(80)$ & $11(55)$ & $11(55)$ & $20(16)$ \\
\hline Sensory urgency & $5(83)$ & $5(83)$ & $2(33)$ & $3(50)$ & $6(5)$ \\
\hline $\begin{array}{l}\text { Bladder outflow } \\
\text { obstruction }\end{array}$ & $7(58)$ & $9(75)$ & $6(50)$ & $3(25)$ & $12(9)$ \\
\hline Hypocontractile & $10(83)$ & $10(83)$ & $6(50)$ & $2(17)$ & $12(9)$ \\
\hline Acontractile & $1(50)$ & $2(100)$ & $2(100)$ & $0(0)$ & $2(2)$ \\
\hline Normal & $2(100)$ & $1(50)$ & $1(50)$ & $1(50)$ & $2(2)$ \\
\hline $\begin{array}{l}\text { Other (vaginal reflux/ } \\
\text { urethral pooling/ } \\
\text { detrusor sphincter } \\
\text { dyssynergia/ reduced } \\
\text { capacity) }\end{array}$ & $8(62)$ & $9(69)$ & $5(38)$ & $5(38)$ & $13(10)$ \\
\hline
\end{tabular}


UDS suite and is relied upon to clearly annotate the recording with event markers. It is essential that patients having aUDS have the cognitive ability to comply with the instructions from their urodynamicist and that the test be performed using a strict protocol with regular review. Following the test, the extensive traces generated must be reviewed by the same urodynamicist, allowing for accurate correlation between the patient's bladder symptoms and urodynamic findings. While aUDS is a time-consuming and expensive test, it has been shown to be well tolerated[15], with $85 \%$ of patients happy to attend for further studies. Following aUDS,18.6\% of patients experience mild to moderate de novo dysuria, and $1.1 \%$ experience asymptomatic bacterial UTI[16].

At our centre, we performed 1461 conventional/ vUDS in the same time period as the 84 aUDS studies, or 1 aUDS to every 17.4 conventional/ vUDS studies. Whilst aUDS is the most accurate urodynamic diagnostic test, it takes 2 to 4 hours to perform and 1 to 2 hours to interpret the results compared with 30 to 60 minutes in total for CMG/vUDS. It is therefore not costor time-effective to perform aUDS on all patients and aUDS should be reserved for patients with significantly bothersome symptoms, who are contemplating invasive treatment and in whom conventional and/or vUDS have been non-diagnostic or contradictory to their symptomatology.

In our study, urodynamic diagnosis was changed in $79 \%$ of our cohort following aUDS. This resulted in a change in clinical diagnosis in $89 \%$ of patients having their urodynamic diagnosis changed following aUDS, whilst a change in clinical diagnosis was made in $70 \%$ of all patients having aUDS. This is similar to the $72 \%$ to $89 \%$ [8-10] of successful clinical diagnosis made following aUDS in previous studies where aUDS traces were evaluable. These changes in urodynamic and subsequent clinical diagnosis allowed treatment to be modified in $83 \%$ of our patient cohort having change in urodynamic diagnosis post aUDS and in $75 \%$ of all patients having aUDS, which is higher than the $43 \%$ [9] to $63 \%$ [8] described in previous studies. The higher treatment change rates noted in our study might be a consequence of patients having aUDS only if they had non-diagnostic or contradictory prior conventional or vUDS before consideration for operative intervention.

We do not perform invasive urodynamic assessment prior to non-operative intervention, as per NICE guidance[2]. It was an unexpected finding and of interest that some patients would have 2 different urodynamic tests to establish a diagnosis based on their wish to have further and more invasive treatment-and then decline this treatment. This is however their prerogative. Our general experience is that while ambulatory urodynamics tests are time-consuming, most patients tolerate this well and all are happy that a correct urodynamic diagnosis can be provided and hence the most appropriate treatment offered.

Within the United Kingdom health care system, invasive treatment options such as intravesical botulinum toxin and sacral neuromodulation are offered only after a proven urodynamic diagnosis of DO. At our centre we do not treat patients who have mild SUI demonstrated on conventional UDS if this does not correlate with their main presenting urinary symptom. Our aUDS results demonstrated DO and UUI in 6 of the 8 patients who had symptomatically contradictory SUI on conventional UDS; therefore, aUDS ensured the patients were treated correctly and prevented any unnecessary surgical intervention for SUI which would not have resolved their predominant urinary symptom. In the $21 \%$ of patients who did not have their urodynamic diagnosis changed following aUDS, a change in clinical diagnosis and management was effected in 56\%. Management was also changed in $50 \%$ of the patients in whom aUDS changed neither urodynamic nor clinical diagnosis. This is most likely due to increased clinician and patient confidence in the urodynamic and clinical diagnosis and hence treatment.

Previous studies have demonstrated satisfactory clinical outcomes in 40\%[9] to $42 \%$ [8] of patients following treatment modification after aUDS. Our higher rates of treatment modification following aUDS resulted in $79 \%$ of our patients having symptomatic improvement following aUDS-initiated changes in diagnosis and treatment. This symptomatic improvement was a statistically significant reduction in day and night frequency, urgency, UUI, SUI, poor flow, strain void, and pain on voiding. Pre-aUDS patients had a median of 4 symptoms (range 2 to 10), and postaUDS patients had a median of 0 symptoms (range 0 to 5). Prior to aUDS, patients were having no or maximal conservative treatment for their "clinical" diagnosis. Our confidence in the post-aUDS urodynamic and clinical diagnosis allowed for effective change in treatment and hence significant symptom improvement.

There are some limitations to this study. Whilst this was a retrospective study, it was of a prospectively acquired urodynamics database and of all consecutive patients having aUDS during our study time period.

\section{Conclusion}

aUDS was able to provide a urodynamic diagnosis in $98 \%$ of patients. This resulted in a change in clinical diagnosis and subsequent change in treatment pathway in $82 \%$ of patients. This study confirms the diagnostic and clinical value of aUDS and is the first to clearly show an $79 \%$ improvement in patient reported symptoms as a consequence. 


\section{References}

1. Rosier P, Schaefer W, Lose G, Goldman HB, Guralnick M, Eustice S, et al. International Continence Society Good Urodynamic Practices and Terms 2016: Urodynamics, uroflowmetry, cystometry, and pressureflow study. Neurourol Urodyn.2017;36(5):1243-1260.

2. Excellence NloC. Urinary incontinence and pelvic organ prolapse in women: management (NG123) 2019. Available at: https://www.nice. org.uk/guidance/ng123.Accessed September 17, 2021.

3. Radley SC, Rosario DJ, Chapple CR, Farkas AG. Conventional and ambulatory urodynamic findings in women with symptoms suggestive of bladder overactivity. J Urol.2001;166(6):2253-2258.

4. Dokmeci F, Seval M, Gok H. Comparison of ambulatory versus conventional urodynamics in females with urinary incontinence. Neurourol Urodyn.2010;29(4):518-521.

5. Digesu GA, Gargasole C, Hendricken C, Gore M, Kocjancic E, Khullar $V$, et al. ICS teaching module: Ambulatory urodynamic monitoring. Neurourol Urodyn.2017;36(2):364-367.

6. Abrams PH, Griffiths DJ. The assessment of prostatic obstruction from urodynamic measurements and from residual urine. Br J Urol.1979; 51(2):129-134.

7. Solomon E, Yasmin H, Duffy M, Rashid T, Akinluyi E, Greenwell TJ. Developing and validating a new nomogram for diagnosing bladder outlet obstruction in women. Neurourol Urodyn.2018;37(1):368-378.

8. Pannek J, Pieper P. Clinical usefulness of ambulatory urodynamics in the diagnosis and treatment of lower urinary tract dysfunction. Scand J Urol Nephrol.2008;42(5):428-432.
9. Gorton E, Stanton S. Ambulatory urodynamics: do they help clinical management? BJOG.2000;107(3):316-319.

10. Cantu H, Sharaf A, Bevan W, Hassine A, Hashim H. Ambulatory urodynamics in clinical practice: A single centre experience. Neurourol Urodyn.2019;38(8):2077-2082.

11. Patravali N. Ambulatory urodynamic monitoring: are we wasting our time? J Obstet Gynaecol.2007;27(4):413-415.

12. Robertson AS, Griffiths C, Neal DE. Conventional urodynamics and ambulatory monitoring in the definition and management of bladder outflow obstruction. J Urol.1996;155(2):506-511.

13. Rademakers KL, Drossaerts JM, Rahnama'i MS, van Koeveringe GA. Differentiation of lower urinary tract dysfunctions: the role of ambulatory urodynamic monitoring. Int J Urol.2015;22(5):503-507.

14. Drossaerts J, Rademakers KLJ, Rahnama'i SM, Marcelissen T, Van Kerrebroeck $P$, van Koeveringe $G$. The value of ambulatory urodynamics in the evaluation of treatment effect of sacral neuromodulation. Urol Int.2019;102(3):299-305.

15. Oh SJ, Son H, Jeong JY, Ku JH. Patients' experience with ambulatory urodynamics. A prospective study. Scand J Urol Nephrol.2006;40(5):391-396.

16. Anders K, Cardozo L, Ashman 0, Khullar V. Morbidity after ambulatory urodynamics. Neurourol Urodyn.2002;21(5):461-463. 